\title{
Comparison of 10 TTP and Tmax Estimation Techniques for MR Perfusion-Diffusion Mismatch Quantification in Acute Stroke
}

\author{
N.D. Forkert, P. Kaesemann, A. Treszl, S. Siemonsen, B. Cheng, H. Handels, J. Fiehler, and G. Thomalla
}

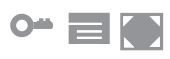

\begin{abstract}
BACKGROUND AND PURPOSE: The mismatch between lesions identified in perfusion- and diffusion-weighted MR imaging is typically used to identify tissue at risk of infarction in acute stroke. The purpose of this study was to analyze the variability of mismatch volumes resulting from different time-to-peak or time-to-maximum estimation techniques used for hypoperfused tissue definition.
\end{abstract}

MATERIALS AND METHODS: Data of 50 patients with middle cerebral artery stroke and intracranial vessel occlusion imaged within 6 hours of symptom onset were analyzed. Therefore, 10 different TTP/Tmax techniques and delay thresholds between +2 and +12 seconds were used for calculation of perfusion lesions. Diffusion lesions were semiautomatically segmented and used for mismatch quantification after registration.

RESULTS: Mean volumetric differences up to 40 and $100 \mathrm{~mL}$ in individual patients were found between the mismatch volumes calculated by the 10 TTP/Tmax estimation techniques for typically used delay thresholds. The application of typical criteria for the identification of patients with a clinically relevant mismatch volume resulted in different mismatch classifications in $\leq 24 \%$ of all cases, depending on the TTP/Tmax estimation method used.

CONCLUSIONS: High variations of tissue-at-risk volumes have to be expected when using different TTP/Tmax estimation techniques. An adaption of different techniques by using correction formulas may enable more comparable study results until a standard has been established by agreement.

ABBREVIATIONS: GVM = $\gamma$-variate model; LDRWM = local density random walk model; LNM = log-normal model; RLCFM = reference-based linear curve fit model; Tmax = time-to-maximum

M ultiparametric MR imaging is currently widely established for diagnosis of patients with acute stroke, ${ }^{1}$ whereas diffusion-weighted and time-resolved perfusion-weighted MR imaging datasets are especially relevant for today's clinical routine. DWI can display ischemic brain tissue with decreased diffusion within minutes from onset. A lesion that is visible in the DWI image sequence is typically assumed to represent the core of an

Received July 11, 2012; accepted after revision November 11.

From the Departments of Computational Neuroscience (N.D.F., P.K.), Medical Biometry and Epidemiology (A.T.), Diagnostic and Interventional Neuroradiology (S.S., J.F.), Neurology (B.C., G.T.), and Institute of Medical Informatics (H.H.), University of Lübeck, Hamburg, Germany.

The research for this paper was funded by the German Research Foundation (grant \# DFG, HA 2355/10-1)

Please address correspondence to Nils Daniel Forkert, MSc, Department of Computational Neuroscience, University Medical Center Hamburg-Eppendorf, Martinistraße 52, 20246 Hamburg, Germany; e-mail: n.forkert@uke.uni-hamburg.de

- - Indicates open access to non-subscribers at www.ajnr.org

$\equiv$ Indicates article with supplemental on-line table.

Indicates article with supplemental on-line figures.

http://dx.doi.org/10.3174/ajnr.A3460 ischemic lesion that is unlikely to recover from therapy. PWI allows identifying hypoperfused tissue resulting from a present vascular occlusion. It has been hypothesized that the volumetric difference between these 2 lesions, the so-called PWI-DWI mismatch, allows a quantitative definition of potentially salvageable brain tissue at risk, which reflects the ischemic penumbra. ${ }^{2-4}$ It is further assumed that a quantification of this mismatch volume allows identifying patients who are likely to benefit from thrombolysis, even in an extended time window after symptom onset. $^{5,6}$ The definition and quantification of the ischemic penumbra is, therefore, increasingly performed for patient selection in randomized controlled trials with treatment beyond the time window that is approved for thrombolytic therapy. ${ }^{7-9}$

Although a Tmax (see below) bolus delay of $>6$ seconds has become an accepted threshold for the definition of a relevant hypoperfusion in recent stroke thrombolysis trials, no official guidelines have been established on how the PWI-DWI mismatch should be calculated. While the identification of acute ischemic lesions on DWI is quite straightforward, the interpretation of PWI is rather challenging. One reason is that several parameters 
can be used for perfusion map calculation. Examples of these parameters comprise the cerebral blood volume, cerebral blood flow, mean transit time, time-to-peak, and time-to-maximum. Several studies have been performed in the past to evaluate the performance of these parameters regarding the ability to predict the final infarct volume, with contradictory outcomes. ${ }^{10,11}$ However, recent trials mainly focus on the time-to-peak and time-tomaximum parameter for PWI-DWI mismatch quantification. For quantitative analyses, the perfusion lesion is typically defined by TTP $>\overline{\mathrm{TTP}}+\tau$ or $\operatorname{Tmax}>\tau$, where $\overline{\mathrm{TTP}}$ describes a mean value within healthy tissue in the contralateral hemisphere and $\tau$, a given temporal delay threshold.

Several studies have been conducted to identify the optimal delay threshold $\tau$ for tissue outcome prediction. However, the findings of these studies are ambiguous, with identified optimal delay thresholds ranging from 4 to 6 seconds, depending on the applied methods and validations. ${ }^{4,12,13}$ Secondary analyses of data from the Echoplanar Imaging Thrombolytic Evaluation Trial (EPITHET) have resulted in a similar refinement of the relevant mismatch criterion by a Tmax delay of $>6$ seconds, which was used in the EXtending the Time for Thrombolysis in Emergency Neurological Deficits (EXTEND) trial. ${ }^{9}$

Several factors may influence the PWI analysis, such as the use and localization of the arterial input function. ${ }^{14,15}$ Apart from this, there is another important aspect of PWI analysis that has not attracted much attention; that is the method for computation of TTP or Tmax parameter maps. Consequently, the method selection may influence the quantitative perfusion lesion definition and subsequent treatment decisions.

The aim of this work was to analyze the impact of different TTP/Tmax estimation techniques on tissue-at-risk quantification at different delay thresholds. It was hypothesized that different hemodynamic models result in relevant differences of tissue-atrisk volumes, with a noticeable effect on the number of patients labeled as exhibiting a relevant PWI-DWI mismatch according to currently used definitions.

\section{METHODS AND MATERIALS \\ Patients and Imaging Protocol}

Fifty nonconsecutive datasets of patients with an acute ischemic stroke in the MCA territory admitted to our hospital since 2004 were analyzed in this study. The selection criterion was a sufficient image quality without movement artifacts so that biased results due to suboptimal image quality could be neglected. Moreover, only patients with an intracranial vessel occlusion identified on time-of-flight MRA were included in this study. The site of vessel occlusion predicts the extent of acute ischemia. ${ }^{16}$ Thus, to include perfusion lesions over the entire distribution range, we selected patients with 4 different occlusion types: carotid-T occlusion; "tandem-occlusion" combining extra- or intracranial ICA with MCA mainstem occlusion (MCA + ICA); MCA mainstem occlusion; and MCA branch occlusion.

All MR imaging measurements were performed on a 1.5T Sonata scanner (Siemens, Erlangen, Germany). The PWI datasets were acquired after application of contrast agent (approximately $15 \mathrm{~mL}$ of gadopentetate dimeglumine [Magnevist; Bayer HealthCare Pharmaceuticals, Wayne, New Jersey]) by using a TR $=1500$ $\mathrm{ms}, \mathrm{TE}=37 \mathrm{~ms}$, and flip angle $=90^{\circ}$; image in-plane image resolution $=0.94 \mathrm{~mm}^{2}$; and 24 sections with 5-mm section thickness. Each acquired PWI dataset contained forty $3 \mathrm{D}$ datasets. The DWI sequences were acquired by applying diffusion gradients in 3 directions with strong diffusion-weighting $\left(b=1000 \mathrm{~s} / \mathrm{mm}^{2}\right.$, $\mathrm{TR}=3500 \mathrm{~ms}, \mathrm{TE}=89 \mathrm{~ms}$, flip angle $=90^{\circ}$; image in-plane image resolution $=0.94 \mathrm{~mm}^{2}$; and 24 sections with 5-mm section thickness).

Informed consent was obtained from all patients. The study was approved by the local ethics committee.

\section{TTP and Tmax Estimation Techniques}

The TTP of a given tissue concentration curve, which can be extracted from each voxel of the PWI dataset, is defined as the time point at which the curve achieves its signal peak. One possibility to calculate this parameter is to determine it directly from the tissueconcentration curves by using the discrete sample points. However, there are 2 main drawbacks when extracting the TTP parameter model independently by using the raw PWI concentration curves: noise artifacts and limited temporal resolution due to the discrete representation.

Thus, hemodynamic model curves are typically used to overcome these limitations. These model curves are fitted to the tissue-concentration curves before TTP estimation. After they are fitted, the adapted model functions can be used for TTP estimation with supposed higher precision.

The following 4 hemodynamic models (Fig 1 ) have been implemented and used for this study: the simplified $\gamma$-variate model, ${ }^{17}$ the local density random walk model, ${ }^{18}$ the modified log-normal model, ${ }^{19}$ and the reference-based linear curve fit model. ${ }^{20}$

The TTP estimation does not account for the shape of the arterial-indicator dilution curves. ${ }^{21}$ Therefore, deconvolutionbased Tmax estimation, which is based on the indicator-dilution theory, ${ }^{22}$ has been proposed as a more precise alternative to direct TTP estimation. The Tmax parameter is defined as the time point at which the residue function $R(t)$ reaches its maximum. Practically, the residue function can be calculated by using the following relation:

$$
C_{T}(t)=\mathrm{CBF} \times R(t) \otimes C_{a}(t),
$$

where $C_{T}(t)$ denotes the tissue concentration curve; $C_{a}(t)$, the arterial input function; $R(t)$, the residue function; $\mathrm{CBF}$, the cerebral blood flow; and $\otimes$, the convolution parameter. Several approaches have been presented in the past that can be used to solve this equation, whereas the singular-value decomposition approach $^{23}$ has been found to achieve good results.

Contrast agent delays among arterial, brain tissue, and venous structures lead to nonidentical recirculation portions in the measured indicator dilution curves because indicator dilution curves are finite. As a result of this, the estimated residue functions may exhibit shape and amplitude errors. ${ }^{24}$ Theoretically, this problem can be solved by fitting of the aforementioned hemodynamic models to the arterial input function and tissue curves before Tmax estimation. 

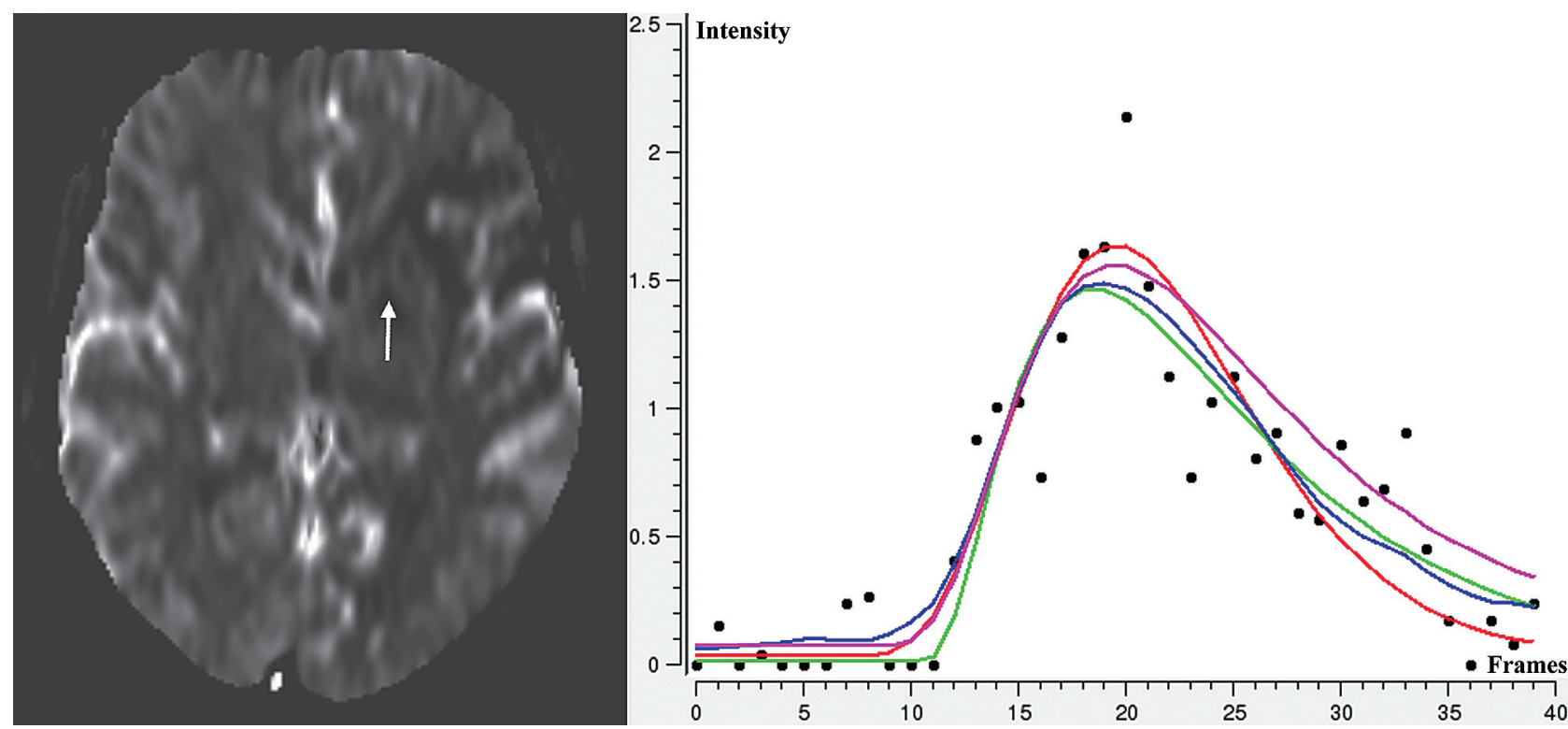

FIG 1. Selected section from a PWI dataset (left) and concentration time curve (black dotted) for a location within the perfusion lesion (arrow) with fitted hemodynamic model curves: $\gamma$-variate model (red), local density random walk model (green), log-normal model (magenta), and reference-based linear curve fit model (blue).

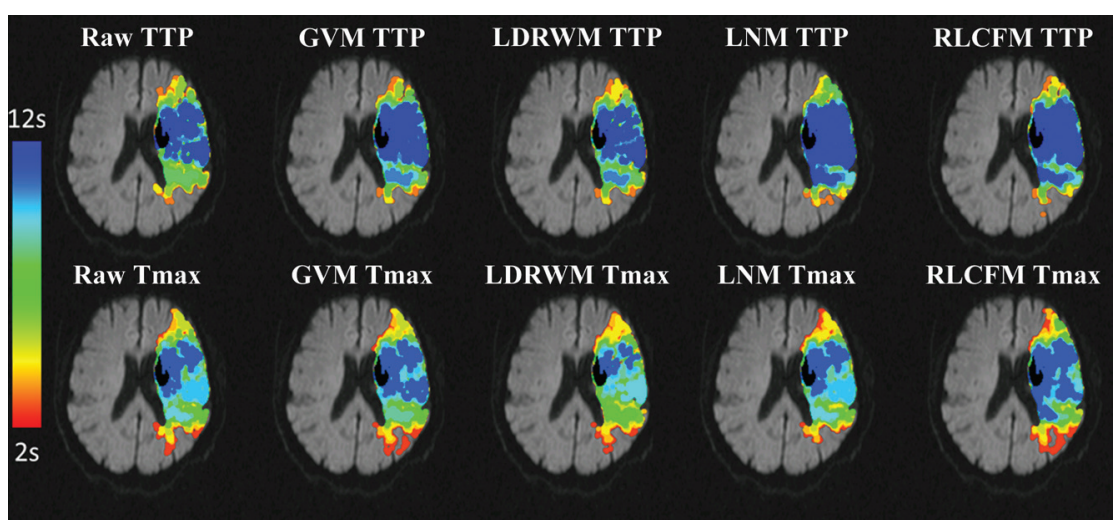

FIG 2. Selected section from a DWI dataset with a corresponding DWI lesion (black) and overlaid tissue-at-risk volumes for delay thresholds between 2 and 12 seconds calculated by using the 10 different TTP/Tmax estimation methods.

\section{Image Processing}

4D PWI datasets display the passage of the paramagnetic contrast agent, which results in a shortening of the $\mathrm{T} 2$ and $\mathrm{T} 2{ }^{*}$ relaxation times and therefore in a reduction of signal intensities. For this reason, the signal functions $S(t)$ need to be converted to concentration curves $C(t)$ to enable an application of the aforementioned TTP/Tmax estimation techniques by using the following formula:

$$
C(t)=-\frac{k}{\mathrm{TE}} \operatorname{In}\left(\frac{S(t)}{S_{0}}\right),
$$

where $S_{0}$ denotes the baseline MR signal intensity; TE, the echo time; and $k$, a proportionality constant.

After conversion, TTP/Tmax perfusion maps were calculated in a voxel wise manner for each PWI dataset by using the mentioned 10 different TTP/Tmax estimation methods (model-independent TTP estimation by using the raw curves, GVM, LDRWM, LNM, and RLCFM as well as model-independent Tmax estimation by using the raw curves, GVM, LDRWM, LNM, and RLCFM).
The arterial input function, required for Tmax estimation, was selected in all datasets from the contralateral middle cerebral artery. The deconvolution, required for Tmax estimation, was performed by using the standard singular value decomposition and a truncation threshold of 0.2 .

For quantification of the PWI-DWI mismatch volume, we manually encircled the visible lesions, including a safety margin at each affected section in the DWI dataset. A second healthy volume of interest was then placed in the contralateral unaffected hemisphere in the corresponding brain tissue, so that the resulting defined volume represented an approximation of the mirrored lesion volume. This healthy volume of interest was then used for calculation of the mean $\mu$ and SD $\sigma$ of the DWI signal intensities, which were then applied for a refinement of the defined coarse DWI lesion volume of interest. Here, all voxels with a signal intensity $I(x)>\mu+2 \sigma$ are retained, while all other voxels are rejected from the final lesion definition.

After this process, the first PWI dataset was registered to the DWI dataset for each patient by using rigid-transformation, linear interpolation, and maximization of the mutual information. The resulting transformation was then used to align the corresponding calculated $10 \mathrm{TTP} / \mathrm{Tmax}$ maps of a patient to the DWI dataset by using a linear interpolation.

After registration, the healthy volume of interest as used for the DWI lesion was also used for determination of the mean TTP or Tmax values for each calculated parameter map, which were used for bolus-delay correction of the TTP and Tmax values. The 
normalization of the Tmax maps was required because the standard singular value decomposition used in this work is sensitive to arterial bolus delay. ${ }^{21}$

These mean values were then applied for automatic PWI lesion definition by using an automatic segmentation method. Here, all voxels within the DWI lesion were used as seeds for a volume-growing by using lower delay thresholds of $\tau=2,3, \ldots, 12$ seconds. For each volume-growing segmentation result, morphologic opening (erosion followed by a dilation) followed by a largest connected component analysis was performed for leakage correction.

Finally, the mismatch volumes were calculated for each TTP/ Tmax estimation technique and delay threshold by voxelwise subtraction of the DWI lesion from the PWI lesion (Fig 2).

All image-processing steps were performed in this study by using the in-house-developed software tool AnToNIa, ${ }^{25}$ which was especially extended to enable all described image-based analyses. Apart from the DWI lesion definition, all mentioned TTP and Tmax estimation methods are implemented in this tool. Furthermore, the registration of the TTP/Tmax maps to the DWI dataset and the subsequent method for PWI-DWI mismatch definition are integrated in the software tool.

\section{Statistics}

Statistical analysis was performed by using the Pearson correlation coefficient to examine the strength of the association between all TTP/Tmax estimation methods for all delay thresholds applied by using the Statistical Package for the Social Sciences (Version 18.0; SPSS, Chicago, Illinois).

For assessment of intermodel TTP/Tmax correction formulas, the analysis distribution of the data was checked and subsequently log-transformed. Random intercept regression analyses (with patient as a random effect) were performed to model one TTP/Tmax parameter on the basis of another. Model-based estimates with 95\% confidence intervals are reported. Nominal $P$ values $<.05$, two-sided, were considered significant. No multiplicity correction was performed. The regression analyses were performed by using SAS 9.2 (SAS Institute, Cary, North Carolina).

As a result of this analysis, a mismatch volume $V_{\mathrm{T}}$ calculated by $1 \mathrm{TTP} / \mathrm{Tmax}$ estimation technique for a given delay threshold $T_{\mathrm{T}}$ can be artificially corrected to the corresponding mismatch volume $V_{\mathrm{R}}$ as calculated by another TTP/Tmax estimation method at the same delay threshold by using the following formula:

$$
V_{R}=\exp \left(A+\log \left(V_{T}\right) \times B+T_{T} \times C\right),
$$

where $A, B$, and $C$ are correction-specific parameters obtained by the random intercept regression analyses.

\section{RESULTS}

\section{Patient Characteristics}

The mean patient age was 66 years (median: 66 years; range: 37-88 years), and the mean time from symptom onset to MR imaging was 154 minutes (median: 127 minutes; range: 60-310 minutes). The infarct was located on the right hemisphere in 22 cases. The median NIHSS score on admission was 15 (range, 3-23).
Ten patients presented a carotid-T occlusion; 10 patients, an ICA+MCA occlusion; 20 patients, an MCA mainstem occlusion; and 10 patients, an MCA branch occlusion.

\section{PWI-DWI Mismatch Volumes}

The mean DWI lesion volume was $26.4 \pm 29.39 \mathrm{~mL}$. The median DWI lesion volume was $19.6 \mathrm{~mL}$ (interquartile range, $8-63 \mathrm{~mL}$ ) for carotid-T occlusions, $13.7 \mathrm{~mL}$ (interquartile range, $10-16 \mathrm{~mL}$ ) for MCA+ICA occlusions, $16.1 \mathrm{~mL}$ (interquartile range, 9-22 $\mathrm{mL}$ ) for MCA mainstem occlusions, and $14.1 \mathrm{~mL}$ (interquartile range, 8-19 $\mathrm{mL}$ ) for MCA branch occlusions.

The results of the PWI-DWI mismatch volumes for the 10 different TTP/Tmax estimation techniques and 11 delay thresholds are illustrated in On-line Fig 2, stratified for the different occlusion types. Overall, the results suggest that the mismatch volumes of all TTP/Tmax estimation techniques are highly correlated because the curve shapes are very similar. Although the general shapes of the resulting curves are very similar, differences regarding the magnitude can be observed. More precisely, the mean volumetric PWI-DWI mismatch differences ranged from only 1.7 to $40.7 \mathrm{~mL}$ (corresponding to percentage differences up to $70 \%$ ) among the results of the $10 \mathrm{TTP} / \mathrm{Tmax}$ estimation methods for the most widely used delay range between $>4$ and $>8$ seconds. In general, Tmax-based mismatch quantification usually led to smaller mismatch volumes compared with the corresponding TTP-based quantification by using the same model (Fig 2).

Stratification of the results for occlusion type revealed the largest mean mismatch volumes for carotid-T occlusions, comparably smaller mismatch volumes for MCA + ICA, isolated MCA mainstem occlusions, and the smallest mismatch volumes for MCA branch occlusions. Within the first 3 mentioned occlusion type groups, differences in mean PWI-DWI mismatch volume ranged between $0.47 \mathrm{~mL}$ and $53.3 \mathrm{~mL}$ for the different estimation methods for delay thresholds between $>4$ and $>8$ seconds. In contrast, MCA branch occlusions presented considerably smaller marked differences, ranging from 0.54 to $25.2 \mathrm{~mL}$ between the average mismatch volumes calculated by the different TTP/Tmax estimation methods.

Patient-specific comparison of individual mismatch volumes determined by the $10 \mathrm{TTP} / \mathrm{Tmax}$ estimation techniques revealed volumetric differences up to $100 \mathrm{~mL}$ for delay thresholds between $>4$ and $>8$ seconds in single patients. Even the most widely used model-independent and GVM TTP/Tmax estimation techniques led to volumetric differences up to $75 \mathrm{~mL}$ within this delay threshold range in individual patients.

\section{Number of Patients with "Relevant" Mismatch}

In decision-making or image-based inclusion criteria of clinical trials, a mismatch considered "relevant" for identifying patients to be treated or not treated is usually defined by the mismatch ratio rather than the plain mismatch volume. Thus, the mismatch volumes were converted to mismatch ratios in a next step. Overall, the graphs for the mismatch ratios stratified for the different occlusion types show the same tendencies as the raw mismatch volumes (On-line Fig 1).

To further relate these findings to clinical decision-making, we calculated the number of patients complying with the penumbral 
inclusion criteria used in the EXTEND trial for each TTP/Tmax estimation method. More precisely, patients exhibiting a PWIDWI mismatch ratio of $>20 \%$ and a PWI-DWI mismatch volume of $>10 \mathrm{~mL}$ at a temporal delay of $>6$ seconds ${ }^{9}$ were considered as presenting a relevant mismatch volume. The results of this evaluation are shown in On-line Fig 3 (top), which displays the number of patients included by each TTP/Tmax estimation technique stratified for each occlusion type. Noticeable discrepancies regarding the number of patients fulfilling these mismatch criteria can be seen. Over the entire sample, the number of patients classified as presenting "no mismatch" ranged from only 3 (RLCFM TTP) to 15 patients (LDRWM TTP and Tmax), depending on the TTP/Tmax estimation method used.

\section{Correlation Analysis and Correction Formulas}

Crosswise correlation analysis of the tissue-at-risk volumes at the different delay thresholds determined by the different TTP/Tmax estimation techniques revealed highly significant strong correlation coefficients, ranging from 0.896 to 0.998 .

These findings support the assumption that the tissue-at-risk volumes derived from the different TTP/Tmax estimation techniques are comparable to some extent and a correction between the models is feasible. Quantitatively, the correction formulas allowed an adaptation with a nonlogarithmized mean standard error of $3.64 \mathrm{~mL}$ (minimum, $0.84 \mathrm{~mL}$; maximum, $7.46 \mathrm{~mL}$ ) over all TTP/Tmax estimation techniques and delay thresholds (On-line Table). In general, a conversion from TTP to Tmax or vice versa led to higher standard errors (mean standard error, $4.28 \mathrm{~mL}$ ) than the conversion between 2 TTP and 2 Tmax estimation methods (mean standard error, $2.96 \mathrm{~mL}$ ). The correction parameters derived from the random intercept regression analyses can be found in the On-line Table.

To investigate a possible clinical benefit associated with these correction formulas, we exemplarily analyzed whether this adaptation is beneficial in terms of reduced differences regarding the definition of patients with a relevant mismatch volume. Therefore, all tissue-at-risk volumes determined by the different TTP/ Tmax estimation methods were corrected to the corresponding mismatch quantification resulting from the model-independent TTP estimation. The results of this evaluation are illustrated in On-line Fig 3 (bottom). Compared with the identification of patients with a relevant PWI-DWI mismatch by using the modelindependent TTP estimation method, differences of up to 7 patients were found for the other TTP/Tmax estimation methods before correction. After correction, maximum differences in patient numbers fulfilling the mismatch criterion decreased to only 2 patients.

\section{DISCUSSION}

The main finding of this study is the observation that different TTP and Tmax estimation methods lead to considerable differences of perfusion lesion volumes and calculated perfusion-diffusion mismatch volumes. In this sample of 50 patients with acute ischemic stroke with different occlusion types, the mean mismatch volumes differed up to $40 \mathrm{~mL}$ among different TTP/Tmax estimation methods. Moreover, for individual patients, the differences in lesion volumes increased up to $100 \mathrm{~mL}$. These findings are novel because there is no previous systematic comparison of different TTP/Tmax estimation methods in patients with acute ischemic stroke.

The PWI-DWI mismatch definition may be influenced by several parameters to different extents, which is beyond the focus of this study. Among others, these parameters comprise the injection protocol (injection volume and injection rate), MR imaging parameters, usage and techniques for preprocessing including deconvolution, perfusion parameter selection, arterial input function localization, image registration accuracy, and finally the definition of thresholds to define critical hypoperfusion. ${ }^{11,14,15,21}$ Although several studies have evaluated the impact of 1 or more of these different parameters directly or indirectly, the influence of the TTP/Tmax estimation technique has not gained much attention. So far, only 1 study has been conducted comparing direct TTP and deconvolution-based Tmax estimation. ${ }^{13}$ With PET as a criterion standard, it was concluded that deconvoluted Tmax does not perform significantly better than the direct TTP estimation. Moreover, the optimal TTP delay threshold was estimated at 4.2 seconds, while the optimal Tmax threshold was determined at 5.5 seconds in this study. This finding also substantiates the conclusions of this work that different TTP and Tmax methods lead to volumetric differences of perfusion lesions when applying inappropriate delay thresholds. However, only model-independent direct TTP and Tmax estimation was evaluated in this PET study, ${ }^{13}$ while the use of hemodynamic model curves was not investigated.

The numerous possible influence factors and the heterogeneity of techniques and parameters pose a vital challenge to acute stroke perfusion image analysis, making direct comparisons between studies that employed different methods complex, if not impossible. ${ }^{26}$ Stroke researchers have addressed this problem by a consensus statement of an Acute Stroke Research Imaging Roadmap. ${ }^{27}$ Nevertheless, a recent systematic review still identified substantial heterogeneity of perfusion image acquisition and postprocessing as well as considerable under-reporting of methodology in scientific publications. ${ }^{26}$ The present study sheds light on an essential part of the postprocessing pipeline that has not been the focus of most stroke imaging researchers until now.

Besides this rather scientific problem, the results of this study also have an immediate clinical implication. The identification of tissue at risk by estimation of perfusion and diffusion lesions is increasingly used to guide treatment decisions in acute stroke. ${ }^{1,5,6}$ While differences in perfusion lesion extent as observed in this study may not make a difference in the case of a large perfusion lesion with a clear mismatch, they may be decisive in borderline cases in which the decision, whether a relevant mismatch is present, is not eye-catching. This possibility applies especially to clinical trials that use a penumbral MR imaging pattern to identify a target population of patients with acute stroke. ${ }^{7-9}$ In these trials, a clear definition of mismatch has to be used, and differences in perfusion lesion quantification, as reported in this study, may determine whether the patients have a mismatch. If one applies the relevant mismatch definition that is currently used in the EXTEND trial, ${ }^{9}$ the TTP/Tmax estimation methods tested in this study resulted in a different mismatch classification in $\leq 24 \%$ of all cases (12/50). 
Especially in the setting of multicenter trials, this difference carries the risk of significant inclusion heterogeneity, which, in the end, may even influence the results of the trial. A number of re-analyses of data from the EPITHET have demonstrated that only slight modifications of the definition of mismatch, such as the use of coregistered images to calculate mismatch volume instead of simple volume subtraction, may change the trial from a negative to a positive one with respect to the primary end point. ${ }^{28}$ Thus, especially in a clinical trial or any study involving multiple sites, standardization of image acquisition and processing represents a key factor to assure homogeneous results.

It was not an aim of this study to identify the optimal TTP/ Tmax estimation technique or the best delay threshold. However, the results of this study revealed that the definition of an optimal delay threshold may only be valid for a certain TTP/Tmax estimation technique.

This study indicates a way to overcome the problem of heterogeneous TTP/Tmax estimation algorithms used in stroke research by calculating correction formulas to convert results from one estimation model into another. Although the results of different studies will still not be directly comparable, a rough adjustment appears possible by using the described correction formulas. However, the correction formulas have only been evaluated exemplarily in this work so that no conclusions can be drawn from this regarding a possible general application. It may be possible that the calculated parameters of the correction formulas depend on the contrast agent injection protocol, PWI acquisition parameters, or localization of the arterial input function. Therefore, further evaluations of the presented correction formulas are required.

A suboptimal fitting of a hemodynamic model curve to the arterial input function may lead to a systematic error for the whole Tmax map calculation. Thus, special care needs to be taken to ensure optimal fitting to the arterial input function in this case.

Apart from this problem, an occluded vessel may lead to considerable perfusion changes so that the concentration curves do not exhibit a typical shape in the worst case. Thus, an optimal model curve fitting may not be possible in certain stroke areas because the underlying assumptions are not valid. The referencebased linear curve fitting approach ${ }^{20}$ recently has been suggested, which differs from the other hemodynamic models in that no explicit assumption about typical concentration curve shape is made, to overcome this problem. The results of this study suggest that this approach leads to bigger mismatch volumes compared with the other TTP/Tmax estimation methods. This finding may be ascribed to better curve fitting in areas that are affected by a severe perfusion disturbance.

Finally, the arterial input function was selected in all datasets from the contralateral middle cerebral artery and was used in a standard singular value decomposition approach for Tmax estimation. Therefore, it may be interesting for further studies to evaluate more sophisticated deconvolution techniques ${ }^{21}$ in combination with varying global or even local arterial input functions.

\section{CONCLUSIONS}

High variations of tissue-at-risk volumes, which may also be clinically relevant, have to be expected when using different
TTP/Tmax estimation techniques. An adaption of different techniques using correction formulas may enable more comparable study results until a standard has been established by agreement.

Disclosures: Nils Daniel Forkert—RELATED: German Research Foundation (Ha2355/ 10-1).* Jens Fiehler-Consulting Fee or Honorarium: Siemens, Comments: Congress presentation on acute stroke imaging. ${ }^{\star}$ Money paid to the institution.

\section{REFERENCES}

1. Muir KW, Buchan A, von Kummer R, et al. Imaging of acute stroke. Lancet Neurol 2006;5:755-68

2. Butcher KS, Parsons M, MacGregor L, et al. Refining the perfusiondiffusion mismatch hypothesis. Stroke 2005;36:1153-59

3. Neumann-Haefelin T, Wittsack HJ, Wenserski F, et al. Diffusionand perfusion-weighted MRI. The DWI/PWI mismatch region in acute stroke. Stroke 1999;30:1591-97

4. Sobesky J, Zaro Weber O, Lehnhardt FG, et al. Which time-to-peak threshold best identifies penumbral flow? A comparison of perfusion-weighted magnetic resonance imaging and positron emission tomography in acute ischemic stroke. Stroke 2004;35:2843-47

5. Thomalla G, Schwark C, Sobesky J, et al. Outcome and symptomatic bleeding complications of intravenous thrombolysis within 6 hours in MRI-selected stroke patients: comparison of a German multicenter study with the pooled data of ATLANTIS, ECASS, and NINDS tPA trials. Stroke 2006;37:852-58

6. Schellinger PD, Thomalla G, Fiehler J, et al. MRI-based and CTbased thrombolytic therapy in acute stroke within and beyond established time windows: an analysis of 1210 patients. Stroke 2007;38:2640-45

7. Hacke W, Furlan AJ, Al-Rawi Y, et al. Intravenous desmoteplase in patients with acute ischaemic stroke selected by MRI perfusiondiffusion weighted imaging or perfusion CT (DIAS-2): a prospective, randomised, double-blind, placebo-controlled study. Lancet Neurol 2009;8:141-50

8. Davis SM, Donnan GA, Parsons MW, et al. Effects of alteplase beyond $3 \mathrm{~h}$ after stroke in the Echoplanar Imaging Thrombolytic Evaluation Trial (EPITHET): a placebo-controlled randomised trial. Lancet Neurol 2008;7:299-309

9. Ma H, Parsons MW, Christensen S, et al. A multicentre, randomized, double-blinded, placebo-controlled phase III study to investigate EXtending the time for Thrombolysis in Emergency Neurological Deficits (EXTEND). Int J Stroke 2012;7:74-80

10. Schaefer PW, Hunter GJ, He J, et al. Predicting cerebral ischemic infarct volume with diffusion and perfusion MR imaging. AJNR Am J Neuroradiol 2002;23:1785-94

11. Kane I, Carpenter T, Chappell F, et al. Comparison of $\mathbf{1 0}$ different magnetic resonance perfusion imaging processing methods in acute ischemic stroke: effect on lesion size, proportion of patients with diffusion/perfusion mismatch, clinical scores, and radiologic outcomes. Stroke 2007;38:3158-64

12. Olivot JM, Mlynash M, Thijs VN, et al. Optimal Tmax threshold for predicting penumbral tissue in acute stroke. Stroke 2009;40:469-75

13. Zaro-Weber O, Moeller-Hartmann W, Heiss WD, et al. Maps of time to maximum and time to peak for mismatch definition in clinical stroke studies validated with positron emission tomography. Stroke 2010;41:2817-21

14. Ebinger M, Brunecker P, Jungehulsing GJ, et al. Reliable perfusion maps in stroke MRI using arterial input functions derived from distal middle cerebral artery branches. Stroke 2010;41:95-101

15. Christensen S, Mouridsen K, Wu O, et al. Comparison of 10 perfusion MRI parameters in 97 sub-6-hour stroke patients using voxelbased receiver operating characteristics analysis. Stroke 2009;40: 2055-61

16. Fiehler J, Knudsen K, Thomalla G, et al. Vascular occlusion sites determine differences in lesion growth from early apparent diffu- 
sion coefficient lesion to final infarct. AJNR Am J Neuroradiol 2005;26:1056-61

17. Madsen MT. A simplified formulation of the gamma variate function. Phys Med Biol 1992;37:1597-600

18. Bogaard JM, Smith SJ, Versprille A, et al. Physiological interpretation of the skewness of indicator-dilution curves; theoretical considerations and a practical application. Basic Res Cardiol 1984;79: 479-93

19. Linton RA, Linton NW, Band DM. A new method of analysing indicator dilution curves. Cardiovasc Res 1995;30:930-38

20. Forkert ND, Fiehler J, Ries T, et al. Reference-based linear curve fitting for bolus arrival time estimation in 4D MRA and MR perfusion-weighted image sequences. Magn Reson Med 2011;65:289-94

21. Kudo K, Sasaki M, Ostergaard L, et al. Susceptibility of Tmax to tracer delay on perfusion analysis: quantitative evaluation of various deconvolution algorithms using digital phantoms. J Cereb Blood Flow Metab 2011;31:908-12

22. Meier P, Zierler KL. On the theory of the indicator-dilution method for measurement of blood flow and volume. J Appl Physiol 1954; 6:731-44

23. Ostergaard L, Weisskoff RM, Chesler DA, et al. High resolution mea- surement of cerebral blood flow using intravascular tracer bolus passages. Part I. Mathematical approach and statistical analysis. Magn Reson Med 1996;36:715-25

24. Straka M, Albers GW, Bammer R. Real-time diffusion-perfusion mismatch analysis in acute stroke. J Magn Reson Imaging 2010; 32:1024-37

25. Thomalla G, Cheng B, Ebinger M, et al. DWI-FLAIR mismatch for the identification of patients with acute ischaemic stroke within $4.5 \mathrm{~h}$ of symptom onset (PRE-FLAIR): a multicentre observational study. Lancet Neurol 2011;10:978-86

26. Dani KA, Thomas RG, Chappell FM, et al. Systematic review of perfusion imaging with computed tomography and magnetic resonance in acute ischemic stroke: heterogeneity of acquisition and postprocessing parameters: a translational medicine research collaboration multicentre acute stroke imaging study. Stroke 2012; 43:563-66

27. Wintermark M, Albers GW, Alexandrov AV, et al. Acute stroke imaging research roadmap. Stroke 2008;39:1621-28

28. Nagakane Y, Christensen S, Brekenfeld C, et al. EPITHET: positive result after reanalysis using baseline diffusion-weighted imaging/ perfusion-weighted imaging co-registration. Stroke 2011;42:59-64 